\title{
Constraining the late heavy bombardment of the Moon using Ru isotopes in lunar impactites
}

\author{
E. A. WORSHAM ${ }^{1}$ AND T. KLEINE ${ }^{1}$
}

${ }^{1}$ Institut für Planetologie, University of Münster, 48149

Münster, Germany (worsham@uni-muenster.de)

The heavily cratered surface of the Moon indicates that a late heavy bombardment (LHB) occurred after the formation of its rigid crust, but the timing, composition, and delivery mechanism of the LHB are not well constrained [e.g., 1]. The impactors involved can be directly investigated using lunar impactites, which formed via the modification of lunar target rocks by large impacts. As siderophile elements are strongly depleted in the Moon's crust due to core formation, but have comparatively high concentrations in projectile materials, impactites are often enriched in siderophile elements inherited from the impactor. The highly siderophile element $\mathrm{Ru}$ is useful because it displays nucleosynthetic isotope anomalies at the bulk meteorite scale [2], which can be used as genetic tracers to characterize the impactors. Ruthenium isotopes can also distinguish the inner solar system non-carbonaceous (NC) reservoir, and the outer solar system carbonaceous (CC) reservoir [3]. Combined, this makes it possible to use $\mathrm{Ru}$ isotopes to link lunar impactors to distinct types of meteorites and to specific areas of the disk from where these impactors originated, which can place important constraints on the nature of the LHB and on material transport in the early solar system.

The first $\mathrm{Ru}$ isotope data from lunar impactites, including an Apollo 16 sample and a lunar meteorite, indicate that the impactor components in them belong to the NC suite. Further, the impactor components are indistinguishable from the $\mathrm{Ru}$ isotopic composition of the bulk silicate earth (which reflects the isotopic composition of the late veneer, as $\mathrm{Ru}$ was added to the mantle by late accretion). Therefore, the lunar impactors were likely from a similar region to the lateaccreted material added to the Earth following the Moon-forming impact. If confirmed by additional data from more lunar impactites, this would provide strong evidence in favor of the accretion tail scenario of the LHB, where impactors were sourced from leftover planetesimals from the main phase of accretion.

[1] Bottke and Norman. 2017. Annu. Rev. Earth Planet. Sci. 45: 619-47. [2] Fischer-Gödde and Kleine 2017. Nature 541: 525-527. [3] Bermingham et al. 2018. EPSL 487: 221-229. 